\title{
Genome-wide copy number variation analysis of hepatitis B infection in a Japanese population
}

\author{
Masataka Kikuchi $\mathbb{1 0}^{1}$, Kaori Kobayashi ${ }^{1,2}$, Nao Nishida ${ }^{3}$, Hiromi Sawai ${ }^{4,5}$, Masaya Sugiyama ${ }^{3}$, Masashi Mizokami ${ }^{3}$, \\ Katsushi Tokunaga ${ }^{4,6}$ and Akihiro Nakaya ${ }^{1,7}$
}

\begin{abstract}
Genome-wide association studies have been performed to identify common genetic variants associated with hepatitis B (HB). However, little is known about copy number variations (CNVs) in HB. In this study, we performed a genomewide CNV analysis between 1830 healthy controls and 1031 patients with HB infection after quality control. Using signal calling by the Axiom Analysis Suite and CNV detection by PennCNV software, we obtained a total of 4494 CNVs across all individuals. The genes with CNVs that were found only in the HB patients were associated with the immune system, such as antigen processing. A gene-level CNV association test revealed statistically significant CNVs in the contactin 6 (CNTN6) gene. Moreover, we also performed gene-level CNV association tests in disease subgroups, including hepatocellular carcinoma patients, liver cirrhosis patients, and HBV carriers, including asymptomatic carriers and patients with HBV-derived chronic hepatitis. Our findings from germline cells suggested that patient-specific CNVs may be inherent genetic risk factors for $\mathrm{HB}$.
\end{abstract}

\section{Introduction}

Hepatitis $B(\mathrm{HB})$ is an infectious disease caused by the $\mathrm{HB}$ virus (HBV). The World Health Organization (WHO) estimated that 257 million people have chronic HBV infection. HBV infection leads to liver inflammation and can subsequently cause hepatocellular carcinoma (HCC) and liver cirrhosis (LC); in particular, HCC mainly occurs in sub-Saharan Africa and Eastern Asia, including Japan ${ }^{1}$.

To identify the inheritance of a predisposition for $\mathrm{HB}$, genome-wide association studies (GWASs) have been performed using germline tissues such as blood cells in Chinese, Korean, and Japanese populations ${ }^{2-8}$. These GWASs have reported that some susceptibility loci are located in human leukocyte antigen (HLA) regions, including HLA-DPB1, HLA-DPA1, and HLA-DQB2. In addition, a GWAS of an Indonesian population revealed

Correspondence: Masataka Kikuchi (kikuchi@gi.med.osaka-u.ac.jp) or

Akihiro Nakaya (nakaya@edu.k.u-tokyo.ac.jp)

'Department of Genome Informatics, Graduate School of Medicine, Osaka

University, Osaka, Japan

${ }^{2}$ Medical Solutions Division, NEC Corporation, Tokyo, Japan

Full list of author information is available at the end of the article that single nucleotide polymorphisms (SNPs) in the HLA regions contributed to the reactivity to the $\mathrm{HB}$ vaccine? HLA presents antigens, including those produced during viral infections, to $\mathrm{T}$ cells and regulates the immune system. This result suggests that immune system variations in individuals are associated with the pathogenesis of HB. However, little is known about the contribution of structural variants such as copy number variations (CNVs) to HB.

Genomic DNA is usually present in two copies in a human cell, whereas its copy number changes by genome duplication and deletion events. These DNA segments differing in copy number in a population are called CNVs. CNVs can affect gene expression and are associated with the development of disease.

In somatic cells, the integration of HBV into the genome of human hepatocytes increases chromosomal instability and has the potential to alter the copy number of human $\mathrm{DNA}^{10}$. Indeed, the number of somatic CNVs in liver tissues of patients with HCC was positively correlated with the number of $\mathrm{HBV}$ integrations into the human genome ${ }^{10}$. Liver tissues from patients with LC 
have more CNVs than those from healthy participants ${ }^{11}$. These studies suggested that the accumulation of somatic $\mathrm{CNV}$ s reflects the severity of the pathology and the degree of HBV integration.

In the germline, Clifford et al. examined CNVs in 386 patients with $\mathrm{HCC}$ in Korean and Chinese populations and identified CNVs in the ALDH7A1, C4orf29, C5orf48, KNG1, LARP2, LMNB1, PHAX, PUS7, SRPK2, and TMPO genes ${ }^{12}$. Some of these CNVs were related to the immune response and tumorigenesis. However, there have been no large-scale studies of germline CNVs in the Japanese population.

In this study, we performed genome-wide CNV analysis of more than 3000 Japanese individuals to detect germline CNVs. Peripheral blood samples were assayed for CNVs using Axiom Genome-Wide ASI 1 Array Plates optimized to detect not only common variants but also rare variants in the East Asian population. Using signal calling by the Axiom Analysis Suite and CNV detection by PennCNV software, we obtained a total of $4494 \mathrm{CNVs}$ across all individuals. We also calculated various statistics between the case and control individuals and between different disease types, including HCC and LC. The CNV data and statistics from this study will serve as a resource for clarifying the genetic predisposition to $\mathrm{HB}$.

\section{Materials and methods \\ Subjects}

Subjects including healthy controls $(n=2097 ; 31.0 \pm$ 12.2 years old, based on 1514 subjects with age data) and HBV patients $(n=1061 ; 57.8 \pm 12.8$ years old, based on 601 subjects with age data) were recruited at 28 multicenter hospitals (liver units with hepatologists) and universities throughout Japan. These subjects were included in previous studies ${ }^{13,14}$. HBV status was determined based on the serological results for $\mathrm{HB}$ surface antigen (HBsAg) and $\mathrm{HB}$ core antibody (anti-HBc) using a fully automated chemiluminescent enzyme immunoassay system (Abbott ARCHITECT; Abbott Japan, Tokyo, Japan, or LUMIPULSE f or G1200; Fujirebio, Inc., Tokyo, Japan). Unrelated and anonymized healthy control samples were collected from Japanese volunteers with/without HBV vaccination. $\mathrm{HBV}$ patients included $\mathrm{HBV}$-positive $\mathrm{HCC}$ patients $(n=488), \mathrm{HBV}$-positive LC patients $(n=46)$, and HBV carriers, including asymptomatic carriers (ASCs) and individuals with HBV-derived chronic hepatitis $(\mathrm{CH})(\mathrm{ASC}+\mathrm{CH}, n=527)$.

\section{Genotyping and quality control}

GWAS genotyping was performed using the Affymetrix Axiom Genome-Wide ASI 1 Array (Thermo Fisher Scientific, Inc., Waltham, MA, USA), which contains 600,307 markers corresponding to common and rare alleles in a consensus East Asian (including Japanese) genome. First, we excluded poor-quality samples and genotyped the markers of the samples that passed quality control (QC) using the Axiom ${ }^{\mathrm{TM}}$ Analysis Suite. The thresholds for QC were the default thresholds in the Axiom $^{\mathrm{TM}}$ Analysis Suite: dish QC threshold $\geq 0.82$; QC call rate threshold $\geq 97 \%$; plate $\mathrm{QC}$ percent samples passed $\geq 95 \%$; and plate QC average call rate $\geq 98.5 \%$. All individuals who passed QC were examined for potential genetic relatedness by calculating identity-by-descent estimates for all possible pairs of individuals in PLINK. We removed one individual with a low QC call rate within each pair according to pi-hat $(>0.4)$. Finally, we obtained 1831 healthy controls and 1042 HBV patients. We identified CNVs using 345,987 markers that satisfied the following thresholds: minor allele frequency $>0.10$; missing call rate $<1 \%$; and Hardy-Weinberg equilibrium $p$-value $>1.0 \times 10^{-6}$.

\section{CNV calls}

$\mathrm{CNV}$ from the samples were called using PennCNV ${ }^{15}$. PennCNV uses the $\log R$ ratio (LRR) value and the $B$ allele frequency (BAF) for each SNP to infer the copy number states of each SNP. LRR indicates a normalized measure of the total signal intensity of the B and A alleles and directly reflects an increase or decrease in the copy number. The BAF shows a normalized measure of the relative signal intensity ratio of the $B$ and $A$ alleles and helps differentiate copy number states (e.g., differentiate copy-neutral loss of heterozygosity regions and normal state regions). PennCNV calculates the probability of observing a particular copy number state by the hidden Markov model (HMM), given the LRR and BAF for each SNP. A population frequency of the $B$ allele (PFB) file and a GC model file were generated from 1831 healthy controls using compile_pfb.pl and cal_gc_snp.pl in PennCNV. An HMM file was provided by Thermo Fisher Scientific, Inc. Only samples with a standard deviation of the $\log \mathrm{R}$ ratio with a normalized intensity $<0.35, \mathrm{~B}$ allele frequency drifting value $<0.01$, and wave factor value between -0.05 and 0.05 were analyzed. Adjacent CNVs separated by a gap of $<20 \%$ of the combined length of the two CNVs were merged until no more gaps of $<20 \%$ existed, and CNVs based on fewer than 5 markers were excluded. In this process, we examined four cutoffs in terms of the number of markers included in a CNV, which were $>5,>10,>15$, and $>20$ markers. Several genomic regions are known to harbor spurious CNV calls. We excluded centromeric regions, telomeric regions, segmental duplication regions, immunoglobulin regions, and repeat-masked regions. These regions were provided by PennCNV (http://penncnv. openbioinformatics.org/en/latest/misc/faq/). The immunoglobulin regions included four regions (chr2:88937989 -89411302, chr14:21159897-22090937, chr14:105065301 
-106352275, and chr22:20715572-21595082). These regions were transformed from the reference genome hg18 to hg19 using the UCSC LiftOver tool (http://genome. ucsc.edu/cgi-bin/hgLiftOver). We also excluded T-cell receptor (TCR) and immunoglobulin heavy (IGH) chain genomic regions from our analyses because these regions undergo V-(D)-J recombination in lymphocytes and can yield somatic $\mathrm{CNVs}$ rather than germline $\mathrm{CNVs}^{16}$. These regions included TCR alpha and delta on chromosome 14 (chr14:22090057-23021075 and chr14:22891537-22935 569 , respectively), beta and gamma on chromosome 7 (chr7:141998851-142510972 and chr7:38279625-38 407656, respectively), and IGH regions on chromosomes 14 and 16 (chr14:106032614-107288051 and chr16:3 3740716-33741266). Individuals of unknown sex were eliminated. After CNV calls, we finally identified 1830 healthy controls and $1031 \mathrm{HBV}$ patients. Only autosomes were analyzed. PennCNV classifies CNV events according to six state definitions: state $1=$ deletion of two copies (copy number: 0), state $2=$ deletion of one copy (copy number: 1 ), state $3=$ two-copy state (copy number: 2 ), state $4=$ two-copy state with loss of heterozygosity (copy number: 2), state $5=$ duplication of one copy (copy number: 3 ), and state $6=$ duplication of two copies (copy number: 4). A copy number of two was considered normal. CNVs with a copy number $>2$ were defined as duplications, while those with a copy number $<2$ were considered deletions. The distribution of CNVs was drawn by the $\mathrm{R}$ package "RIdeogram"17.

\section{Statistical tests for global burden and gene-level CNV association analyses}

Statistics were calculated by permutation tests $(50,000$ random permutations for the global burden test and 10,000 random permutations for the gene-level CNV association test). The permutation tests were performed using the following procedures: (i) the case-control label of each subject was shuffled, and (ii) the empirical $p$-value of the $j$ th marker was calculated as $(R+1) /(N+$ 1 ), where $N$ is the number of permutations and $R$ is the number of times the permuted test statistics (i.e., $S_{j, 1} \sim S_{j \text {, }}$ $\left.{ }_{N}\right)$ were greater than the observed statistics of the $j$ th marker $S_{j, \text { obs. }} P$-values were adjusted by the $\max (\mathrm{T})$ procedure to regulate the familywise error rate $^{18}$. The $\max (\mathrm{T})$ procedure was performed as follows: (i) the largest statistic of the $j$ th marker $S_{j, \max }$ was selected from the statistics of the permuted data sets, and (ii) an adjusted $p$-value was calculated as $(R+1) /(M+1)$, where $M$ is the number of markers and $R$ is the number of times the permuted test statistics (i.e., $S_{1, \max } \sim S_{M \text {, max }}$ ) were greater than $S_{j \text {,obs. }}$. For gene-level CNV association tests, the gene region was used for UCSC-known gene annotation (knownGene.txt.gz and kgXref.txt.gz). Each test was performed by PLINK software (PLINK command for the global burden test: --cnv-indiv-perm --mperm 50000; PLINK command for the gene-level CNV association test: --cnv-intersect genelist --cnv-testregion --mperm 10000$)^{19}$. In this study, we defined a nonadjusted $p$-value $<0.05$ as nominally significant.

\section{Gene functional enrichment analysis}

We used the Molecular Signatures Database (MSigDB) in Gene Set Enrichment Analysis (GSEA) and Metascape software to examine the functions of genes ${ }^{20-22}$. We examined overlaps with Gene Ontology (GO) biological processes in the GSEA-MSigDB analysis. HBV patient-unique genes were defined as genes with one or more CNV in HBV patients and no CNVs in the healthy controls. In contrast, the healthy control-unique genes were defined as the genes found to have more than one $\mathrm{CNV}$ in the healthy controls and none found in the HBV patients.

\section{Genomic regulatory regions for 127 tissues or cell types}

Genomic regulatory regions for 127 tissues or cell types were obtained from the Roadmap Epigenomics website (http://egg2.wustl.edu/roadmap/web_portal/). The chromatin state model segments the human genome into 25 states based on 12 chromatin marks (H3K4me1, H3K4me2, H3K4me3, H3K9ac, H3K27ac, H4K20me1, H3K79me2, H3K36me3, H3K9me3, H3K27me3, H2A.Z, and DNase I-hypersensitive sites) using ChromHMM and ChromImpute ${ }^{23,24}$. We grouped these states into the following 12 subgroups: active TSS (TssA), promoter (PromU, PromD1, and PromD2), transcribed (Tx5, Tx, Tx3, and TxWk), transcribed and regulatory (TxReg, TxEnh5, TxEnh3, and TxEnhW), active enhancer (EnhA1, EnhA2, and EnhAF), weak enhancer (EnhW1, EnhW2, and EnhAc), DNase (DNase), ZNF genes and repeats (ZNF/Rpts), heterochromatin (Het), poised promoter (PromP), bivalent promoter (PromBiv), and repressed polycomb (ReprPC). The DNA segments in these 12 subgroups from each tissue or cell type are described in bed files. We compared the number of genomic regulatory regions included in the CNVs between the healthy controls and the HBV patients with the following PLINK command: --cnv-indiv-perm --mperm 50000 --cnv-count bedfile.

\section{Results}

\section{Global CNV burden analysis}

We performed global CNV burden analysis using four different $\mathrm{CNV}$ cutoffs to examine the characteristics of CNVs between 1830 healthy controls and $1031 \mathrm{HBV}$ patients. We detected $2098 \mathrm{CNVs}$ with a cutoff of $>20$ markers and $4470 \mathrm{CNVs}$ with a cutoff of $>5$ markers in the healthy controls and HBV patients (Fig. 1, Table 1). 


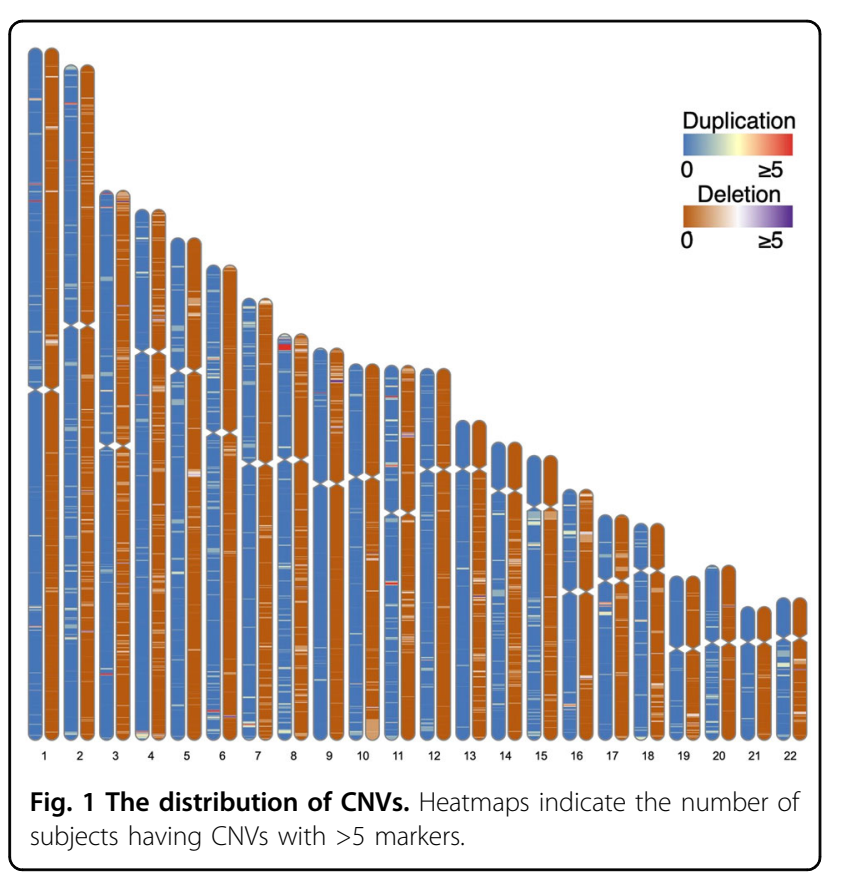

The proportion of samples with one or more CNV in HBV patients (74.6\%) was higher than that in the healthy controls $(68.5 \%)$ for a cutoff of $>5$ markers (adjusted $p$-value $\left.=1.84 \times 10^{-3}\right)$, although there was no significant difference for the other cutoffs (Table 1). The number of CNVs with at least one gene in the HBV patients (0.552) was higher than that in the healthy controls $(0.498)$ for a cutoff of $>5$ markers (adjusted $p$-value $=1.27 \times 10^{-2}$ ). This difference was not significant for the other cutoffs, suggesting that this tendency depended on the proportion of samples with one or more CNV.

We identified 1153 genes with CNVs for a cutoff of $>5$ markers in the HBV patients. Among these genes, 751 were unique to the HBV patients. We next performed gene functional enrichment analysis using GSEA software to identify the functions of these HBV patient-unique genes with CNVs. As a result, 22q11.2 deletion syndrome (falsediscovery rate $(\mathrm{FDR})$-adjusted $p$-value $=6.82 \times 10^{-27}$ ), steroid hormone biosynthesis (FDR-adjusted $p$-value $=$ $\left.4.54 \times 10^{-4}\right)$, and antigen processing and presentation (FDR-adjusted $p$-value $=1.62 \times 10^{-3}$ ) were significantly associated with the HBV patient-unique genes (Table S1). Metascape software also revealed these associations (FDRadjusted $p$-value $<0.05$ ) (Table S2). Interestingly, the genes with CNVs found in the healthy controls were not associated with these terms (Tables S3 and S4).

\section{Genomic regulatory regions covered by CNVs}

We next focused on the genomic regulatory regions activated in different tissues and investigated those included in the CNVs in the HBV patients. The number of genomic

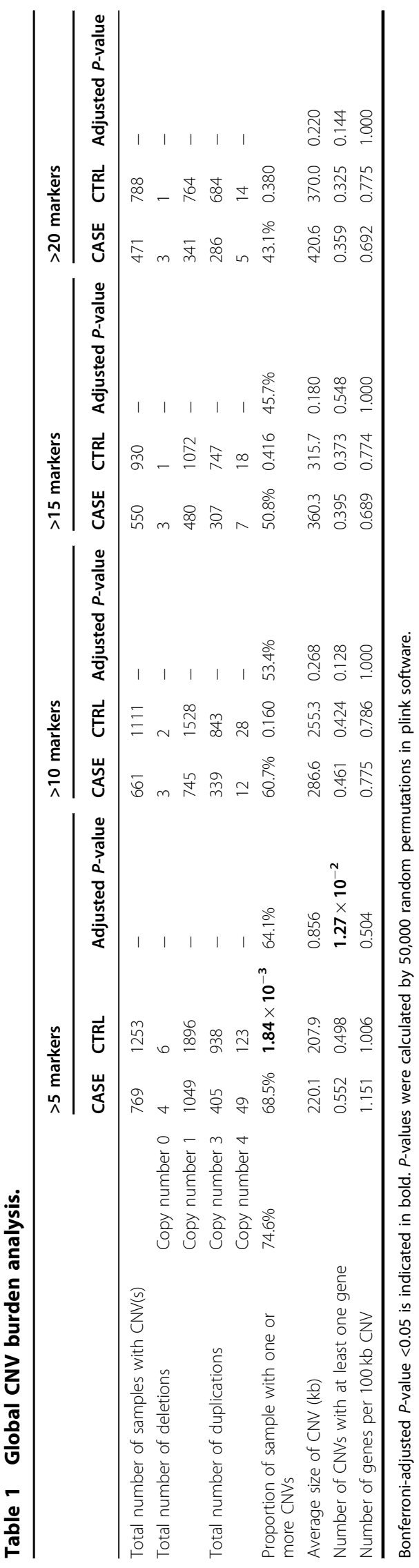




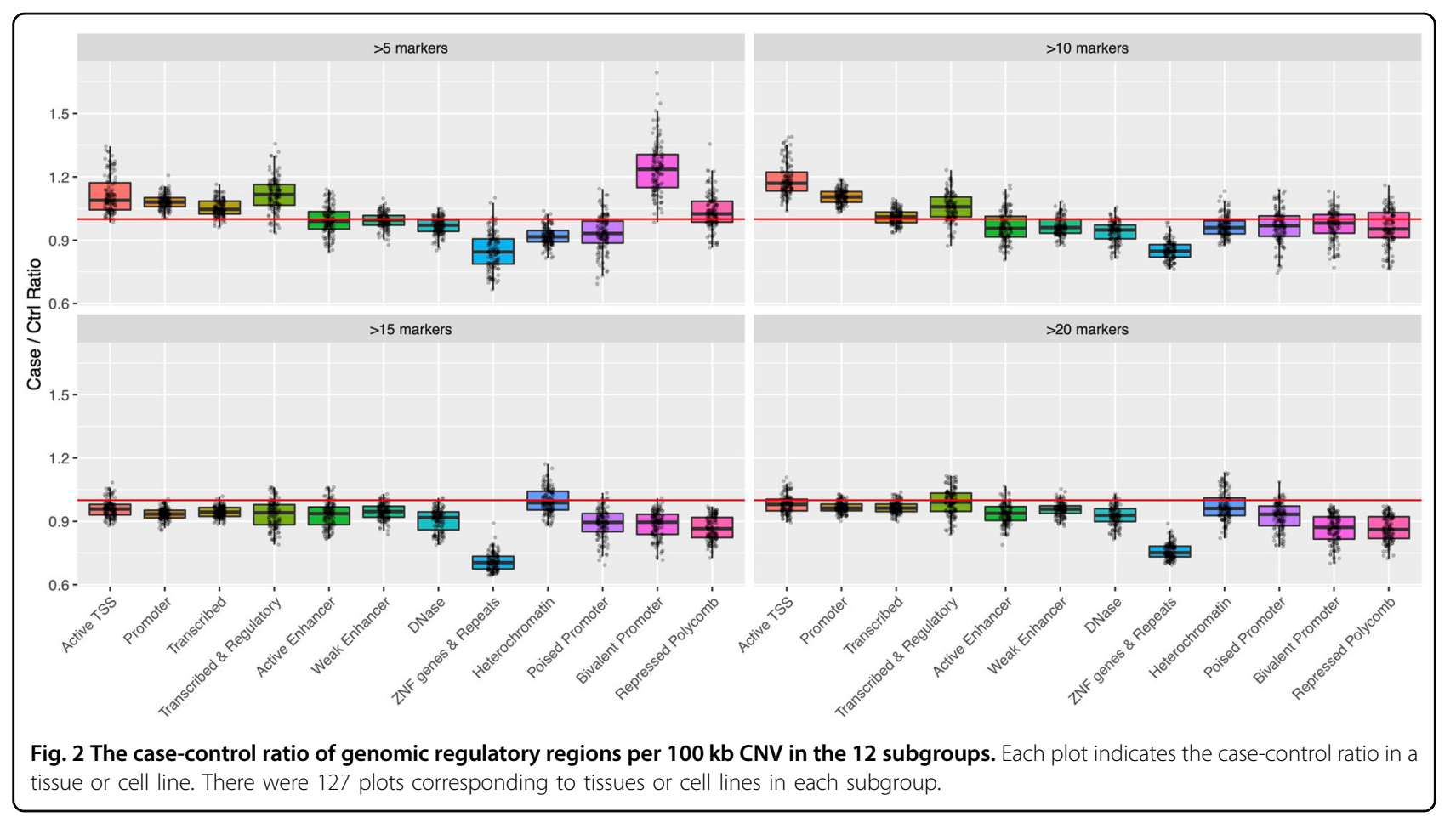

regulatory regions included in the CNVs was compared between the healthy controls and the HBV patients using a chromatin state model for 127 tissues or cell types from Roadmap Epigenomics. We divided the genomic regulatory regions into 12 subgroups (see the "Materials and methods" section). Figure 2 shows the number of genomic regulatory regions per $100 \mathrm{~kb} \mathrm{CNV}$ in each subgroup as the ratio between the HBV patients and the healthy controls. With a cutoff of $>5$ markers, the CNVs in the HBV patients were primarily dominated by six subgroups, including promoters and transcribed regions, compared with those in the healthy controls (active TSS, promoter, transcribed, transcribed \& regulatory, bivalent promoter, and repressed polycomb in Fig. 2). Some trends were also observed with a cutoff of $>10$ markers but not with cutoffs of $>15$ and $>20$ markers, indicating that $\mathrm{CNVs}$ with a relatively small size covered the promoter and transcribed regions. As expected, with a cutoff of $>5$ markers, CNVs in the HBV patients nominally significantly covered the TSSs and promoters activated in some cell types in our sample, peripheral blood, compared with the healthy controls, although the FDR-adjusted $p$-values did not reach significance (nonadjusted $p$-value $<0.05$ ) (Table S5).

\section{Gene-level CNV association test}

To search for CNVs associated with HBV, we performed a gene-level $\mathrm{CNV}$ association test between the healthy controls and the HBV patients. We found a significant association between $\mathrm{HB}$ and the $\mathrm{CNVs}$ in the contactin 6
(CNTN6) gene for all cutoffs (adjusted $p$-value < 0.05) (Fig. 3 and S1, Table 2). We also found 13 genes that were nominally associated with HBV with at least one cutoff level (nonadjusted $p$-value $<0.05$ ). All CNV regions were found in the HBV patients rather than in the healthy controls. Therefore, the odds ratios (ORs) were positive only when calculatable.

We next analyzed the CNVs in each disease subgroup. The HBV patients were divided into three disease subgroups: $\mathrm{HCC}, \mathrm{LC}$, and $\mathrm{ASC}+\mathrm{CH}$ patients. $\mathrm{CNVs}$ with nominally significant differences for at least one cutoff level were located in 27, 42, and 11 genes in HCC, LC, and $\mathrm{ASC}+\mathrm{CH}$ patients, respectively (Tables $\mathrm{S} 6-\mathrm{S} 8$ ). The CNVs in CNTN6, which were significant in the above $\mathrm{CNV}$ association test when using all $\mathrm{HBV}$ patients, were found in four patients each from the HCC and ASC $+\mathrm{CH}$ patient subgroups (Tables $\mathrm{S} 6$ and $\mathrm{S} 8$ ). After using 146 age-matched controls aged more than 50 years old $(58.1 \pm 6.45$ years old in the healthy controls and $57.8 \pm 12.8$ years old in the HBV patients), these CNVs were found only in the patients and not in the controls, but the difference was not statistically significant (Table 3).

We finally compared the CNVs between the three disease subgroups: $\mathrm{HCC}$ vs. LC patients, $\mathrm{HCC}$ vs. ASC $+\mathrm{CH}$ patients, and $\mathrm{LC}$ vs. ASC $+\mathrm{CH}$ patients. We did not find significant associations in the comparisons of $\mathrm{HCC}$ vs. $\mathrm{ASC}+\mathrm{CH}$ and $\mathrm{LC}$ vs. $\mathrm{ASC}+\mathrm{CH}$ patients. However, in the comparison of HCC vs. LC patients, the CNVs in 



Fig. 3 Point density plots of the log R ratio and B allele frequency for duplications (A) and deletions (B) of the CNTN6 gene. Each duplication shows low LRR (upper panel), and BAF values clustered around 0 (AAA), 0.33 (AAB), 0.66 (ABB), and 1 (BBB) (bottom panel). We did not find 4-copy duplications in this region. Each deletion shows low LRR (upper panel), and BAF values clustered around 0 or 1 (bottom panel).

collectin 10 (COLEC10) were nominally significant with a cutoff of $>5$ markers (Table S9).

\section{Discussion}

In this study, we examined germline CNVs in Japanese HBV patients. We found that the CNVs in CNTN6 were associated with the HBV patients. To the best of our knowledge, our study examines germline CNVs in HBV patients using the most large-scale genomic dataset in the Japanese population.

We showed that the CNVs in the HBV patients tended to accumulate in genes that play a role in immune system function, such as antigen processing, although we excluded TCR and IGH chain genomic regions, which are regions that dynamically change DNA structure in response to several pathogens (see "Materials and methods") (Tables S1 and S2). The term antigen processing included the following genes: HLA-A, HLA-DRA, PSMB8, TAP1, and TAP2. The PSMB8, TAP1, and TAP2 genes were located near each other on chromosome 6 and were within the same $\mathrm{CNV}$, which may be significant. Our findings suggest that the immune system is potentially impaired in some HBV patients. Figure 2 shows that the CNVs in the HBV patients were included in promoters and transcribed regions, suggesting that they directly affect the expression levels of genes (e.g., those in the immune system).

In the gene-level $\mathrm{CNV}$ association test of the HBV patients, the CNV region covering CNTN6 was statistically significant across all cutoffs (Table 2). CNTN6 belongs to the immunoglobulin superfamily and is implicated in neural developmental events, including neural cell adhesion and migration, neurite outgrowth and fasciculation, axon guidance, and myelination ${ }^{25-27}$. A study showed that HBV DNA integrated into CNTN6 in HBV-related HCC tissues ${ }^{28}$.

In the gene-level $\mathrm{CNV}$ association test between HCC and LC patients, we detected COLEC10 with a cutoff of $>5$ markers (Table S9). COLEC10 is also called collectin liver 1 (CL-L1) and is mainly expressed in the liver. Members of the collectin family, which belongs to the lectin complement pathway, recognize ligands on pathogens, including viruses and bacteria, and drive phagocytosis. We detected duplication of COLEC10 in two LC patients, suggesting the upregulation of COLEC10 (Table S9). A study reported that the protein levels of COLEC10 in the blood of patients with acute liver failure and alcoholic cirrhosis were higher than those in the blood of healthy controls ${ }^{29}$.

Our study has several limitations. First, we detected statistically significant CNVs in CNTN6, but their associations were not significant after using the agematched controls. Second, most of the CNVs that we found were observed in only a few patients, leading to small effect sizes. Indeed, the 95\% confidence interval of most CNVs had a broad range (Table 2). Third, the sample size of the LC patients was extremely small compared with those of the $\mathrm{HCC}$ and $\mathrm{ASC}+\mathrm{CH}$ patients. To overcome these limitations, we must collaborate with international institutes and validate 

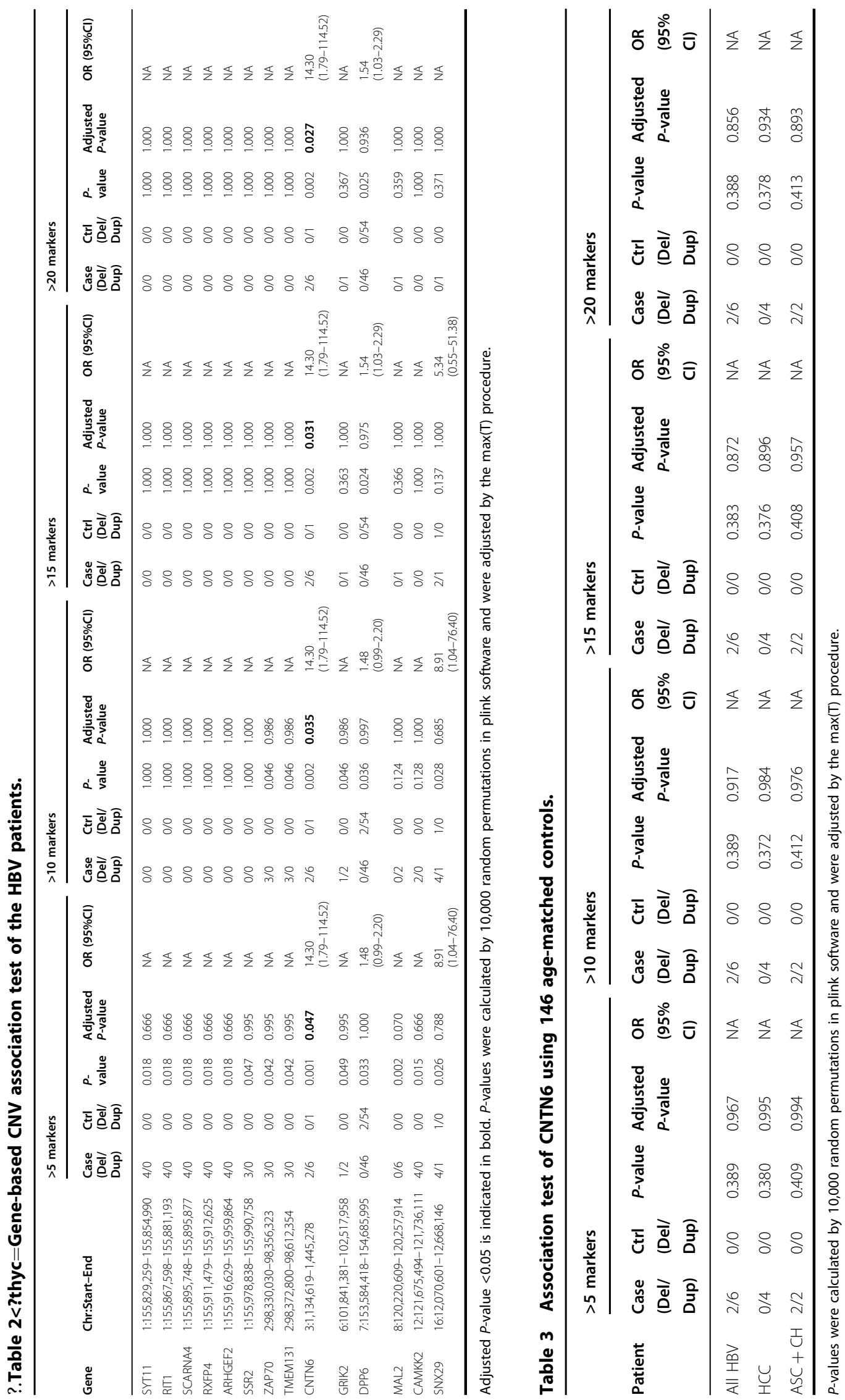


\section{and replicate our findings using more age-matched samples.}

\section{Acknowledgements}

We thank the contributors to sample collection, including Prof. Yasuhito Tanaka (Nagoya City University Hospital), Dr. Kazumoto Murata (National Center for Global Health and Medicine), Prof. Kazuyuki Suzuki (Morioka University), Prof. Yoshikazu Murawaki (Tottori University), Prof. Shuhei Nishiguchi (Hyogo College of Medicine), and Prof. Masaaki Watanabe (Kitasato University Medical Center). This work was supported by Grants-in-Aid for Scientific Research under Grant numbers 17K15049 and 20K15778 to M.K. and $19 K 06629$ to A.N. from the Ministry of Education, Culture, Sports, Science and Technology (MEXT) and by AMED under Grant Number JP16fk0210304 to N.N. and K.T., JP19fk021056h0001 to M.M., K.T., N.N., and M.S., and JP20fk0210056 to N.N., M.S., M.M., and K.T. The funders had no role in the study design, data collection and analysis, decision to publish, or preparation of the manuscript.

\begin{abstract}
Author details
'Department of Genome Informatics, Graduate School of Medicine, Osaka University, Osaka, Japan. ${ }^{2}$ Medical Solutions Division, NEC Corporation, Tokyo, Japan. ${ }^{3}$ Genome Medical Science Project, National Center for Global Health and Medicine, Tokyo, Japan. ${ }^{4}$ Department of Human Genetics, Graduate School of Medicine, The University of Tokyo, Tokyo, Japan. ${ }^{5}$ Present address: Japanese Red Cross Society, Tokyo, Japan. ${ }^{6}$ Present address: Genome Medical Science Project, National Center for Global Health and Medicine, Tokyo, Japan. ${ }^{7}$ Present address: Laboratory of Genome Data Science, Graduate School of Frontier Sciences, The University of Tokyo, Tokyo, Japan
\end{abstract}

\section{Ethical approval}

This study was approved by the Ethics Committee of Osaka University and by all of the following institutes and hospitals throughout Japan that participated in this collaborative study: National Center for Global Health and Medicine, Kawasaki Medical School, Kanazawa University Graduate School of Medicine, National Nagasaki Medical Center, Chiba University, Musashino Red Cross Hospital, Nagoya City University Graduate School of Medical Sciences, Teine Keijinkai Hospital, Shinshu University School of Medicine, Hokkaido University, Saga Medical School, Hokkaido University Graduate School of Medicine, Okayama University Graduate School of Medicine, Osaka City University Graduate School of Medicine, Yamaguchi University Graduate School of Medicine, Kyoto Prefectural University of Medicine, Tottori University, Saitama Medical University, National Hospital Organization Osaka National Hospital, Iwate Medical University, Kurume University School of Medicine, Ehime University Graduate School of Medicine, Hyogo College of Medicine, and Kitasato University School of Medicine. All participants provided written informed consent for participation in this study, and the methods were carried out in accordance with the approved guidelines.

\section{Conflict of interest}

The Department of Genome Informatics is a joint research department established with sponsorship by the NEC Corporation. K.K. is an employee of the NEC Corporation. The funders (NEC Corporation) provided support in the form of salaries for one author (K.K.) but did not have any additional roles in the study design, data collection and analysis, decision to publish, or preparation of the manuscript. All other authors declare no competing interests.

\section{Publisher's note}

Springer Nature remains neutral with regard to jurisdictional claims in published maps and institutional affiliations.

Supplementary information The online version contains supplementary material available at https://doi.org/10.1038/s41439-021-00154-w.

Received: 18 December 2020 Revised: 24 March 2021 Accepted: 18 April 2021.

Published online: 8 June 2021

\section{References}

1. Yang, J. D. et al. A global view of hepatocellular carcinoma: trends, risk, prevention and management. Nat. Rev. Gastroenterol. Hepatol. 16, 589-604 (2019).
2. Kamatani, Y. et al. A genome-wide association study identifies variants in the HLA-DP locus associated with chronic hepatitis B in Asians. Nat. Genet. 41, 591-595 (2009).

3. Mbarek, H. et al. A genome-wide association study of chronic hepatitis B identified novel risk locus in a Japanese population. Hum. Mol. Genet. 20, 3884-3892 (2011).

4. Liu, L. et al. A genome-wide association study with DNA pooling identifies the variant rs11866328 in the GRIN2A gene that affects disease progression of chronic HBV infection. Viral Immunol. 24, 397-402 (2011).

5. Nishida, N. et al. Genome-wide association study confirming association of HLA-DP with protection against chronic hepatitis B and viral clearance in Japanese and Korean. PLoS ONE 7, e39175 (2012).

6. Kim, Y. J. et al. A genome-wide association study identified new variants associated with the risk of chronic hepatitis B. Hum. Mol. Genet. 22, 4233-4238 (2013).

7. $\mathrm{Hu}$, Z. et al. New loci associated with chronic hepatitis B virus infection in Han Chinese. Nat. Genet. 45, 1499-1503 (2013).

8. Cheong, H. S. et al. Association of VARS2-SFTA2 polymorphisms with the risk of chronic hepatitis B in a Korean population. Liver Int. 35, 1934-1940 (2015).

9. Png, E. et al. A genome-wide association study of hepatitis $B$ vaccine response in an Indonesian population reveals multiple independent risk variants in the HLA region. Hum. Mol. Genet. 20, 3893-3898 (2011).

10. Sung, W. K. et al. Genome-wide survey of recurrent HBV integration in hepatocellular carcinoma. Nat. Genet. 44, 765-769 (2012).

11. Brunner, S. F. et al. Somatic mutations and clonal dynamics in healthy and cirrhotic human liver. Nature 574, 538-42. (2019).

12. Clifford, R. J. et al. Genetic variations at loci involved in the immune response are risk factors for hepatocellular carcinoma. Hepatology $\mathbf{5 2}$, 2034-2043 (2010).

13. Nishida, N. et al. Understanding of HLA-conferred susceptibility to chronic hepatitis B infection requires HLA genotyping-based association analysis. Sci. Rep. 6, 24767 (2016).

14. Sawai, H. et al. Genome-wide association study identified new susceptible genetic variants in HLA class I region for hepatitis B virus-related hepatocellular carcinoma. Sci. Rep. 8, 7958 (2018).

15. Wang, K. et al. PennCNV: an integrated hidden Markov model designed for high-resolution copy number variation detection in whole-genome SNP genotyping data. Genome Res. 17, 1665-1674 (2007).

16. Reid, B. M. et al. Genome-wide analysis of common copy number variation and epithelial ovarian cancer risk. Cancer Epidemiol. Biomark. Prev. 28, 1117-26. (2019).

17. Hao, Z. et al. Rldeogram: drawing SVG graphics to visualize and map genomewide data on the idiograms. PeerJ Computer Sci. 6, e251 (2020).

18. Westfall, P. H. \& Young, S. S. Resampling-based Multiple Testing: Examples and Methods for P-value Adjustment (Wiley, 1993).

19. Purcell, S. et al. PLINK: a tool set for whole-genome association and population-based linkage analyses. Am. J. Hum. Genet. 81, 559-575 (2007).

20. Subramanian, A. et al. Gene set enrichment analysis: a knowledge-based approach for interpreting genome-wide expression profiles. Proc. Natl Acad. Sci. USA 102, 15545-15550 (2005).

21. Liberzon, A. et al. The Molecular Signatures Database (MSigDB) hallmark gene set collection. Cell Syst. 1, 417-425 (2015).

22. Zhou, Y. et al. Metascape provides a biologist-oriented resource for the analysis of systems-level datasets. Nat. Commun. 10, 1523 (2019).

23. Ernst, J. \& Kellis, M. ChromHMM: automating chromatin-state discovery and characterization. Nat. Methods 9, 215-216 (2012).

24. Ernst, J. \& Kellis, M. Large-scale imputation of epigenomic datasets for systematic annotation of diverse human tissues. Nat. Biotechnol. 33, 364-376 (2015).

25. Falk, J., Bonnon, C., Girault, J. A. \& Faivre-Sarrailh, C. F3/contactin, a neuronal cell adhesion molecule implicated in axogenesis and myelination. Biol. Cell 94, 327-334 (2002).

26. Karagogeos, D. Neural GPI-anchored cell adhesion molecules. Front. Biosci. 8, s1304-s1320 (2003).

27. Shimoda, Y. \& Watanabe, K. Contactins: emerging key roles in the development and function of the nervous system. Cell Adh. Migr. 3, 64-70 (2009).

28. Ding, D. et al. Recurrent targeted genes of hepatitis $B$ virus in the liver cancer genomes identified by a next-generation sequencing-based approach. PLoS Genet. 8, e1003065 (2012).

29. Laursen, T. L. et al. Circulating mannan-binding lectin, $M-$, L-, H-ficolin and collectin-liver-1 levels in patients with acute liver failure. Liver Int. 35, 756-763 (2015). 